\title{
Systems analysis of the genes responding to chilling stress in Arabidopsis thaliana L.
}

\author{
A. Zlobin ${ }^{1,2}$, N. Omelyanchuk ${ }^{1}$, V. Mironova ${ }^{1,2 *}$ \\ ${ }^{1}$ Institute of Cytology and Genetics SB RAS, Novosibirsk, Russia \\ ${ }^{2}$ Novosibirsk State University, Novosibirsk, Russia \\ *e-mail: victoria.v.mironova@gmail.com
}

Key words: cold acclimation, chilling resistance, cold stress

Motivation and Aim: The chilling resistance of plants, unlike frost resistance, consists in experiencing low positive temperatures, at which ice does not form in the cell, but which affect the reduction of both yield and resistance to other unfavorable factors including pathogens, nutrient deficiencies, high light intensity, etc. Thus, the response to cold affects many processes in the plant, despite the fact that it is triggered by one factor. The objective of this project is the search for and systems analysis of genes that provide a response to low positive temperatures and resistance to them in Arabidopsis. The focus of the project is the mechanisms of chilling resistance in plant roots.

Methods and Algorithms: In our analysis we used more than 20 different datasets on study of chilling stress in Arabidopsis thaliana taken from public access. We preprocessed the datasets uniformly, applying free software environment R. For each dataset we used Benjamini-Hochberg correction, FDR-adjusted p-value $<0.05$ and received list of differentially expressed genes (DEGs). Datasets with less than 50 DEGs were excluded from the analysis.

Results: As a result of meta-analysis of the datasets, we composed a summary table for genes responding to chilling stress in Arabidopsis. More than $3 / 4$ of Arabidopsis genome significantly changed the level of transcription in response to chilling stress at least in one experiment. We identified the genes which systematically changed the level of transcription (in five or more datasets). Six genes we found at the gene network core, among them only SUS1 gene was discovered earlier as cold sensitive in Arabidopsis.

Conclusion: We performed systems analysis of whole-genome experiments on the study of chilling induced changes in transcriptomes. Meta-analysis of these data allowed us reconstructing the gene network of the plant response to chilling stress, description of its functional modules and identification of the feedbacks.

Acknowledgements: The work was supported by RSF 18-14-00249. 